\title{
Preliminary performance measurements of bolometers for the Planck high-frequency instrument
}

Warren Holmes, James J. Bock, Ken Ganga, Viktor Hristov, Leonard Hustead, et al.

Warren Holmes, James J. Bock, Ken Ganga, Viktor Hristov, Leonard Hustead, Timothy Koch, Andrew E. Lange, Chris Paine, Minhee Yun, "Preliminary performance measurements of bolometers for the Planck highfrequency instrument," Proc. SPIE 4855, Millimeter and Submillimeter Detectors for Astronomy, (17 February 2003); doi: 10.1117/12.459421 Hawai'i, United States 


\title{
Preliminary Performance Measurements of Bolometers for the Planck High Frequency Instrument
}

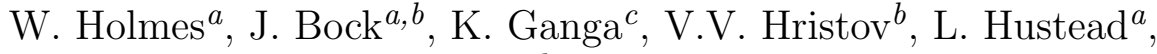 \\ T. Koch ${ }^{a}$, A.E. Lange ${ }^{b}$, C. Paine ${ }^{a}$, and M. Yun ${ }^{a}$ \\ ${ }^{a}$ Jet Propulsion Laboratory, 4800 Oak Grove Dr., Pasadena, CA, USA \\ ${ }^{b}$ Department of Physics, California Institute of Technology, Pasadena, CA, USA \\ ${ }^{c}$ IPAC, California Institute of Technology, Pasadena, CA, USA
}

\begin{abstract}
We report on the characterization of bolometers fabricated at the Jet Propulsion Laboratory for the High Frequency Instrument (HFI) of the joint ESA/NASA Herschel/Planck mission to be launched in 2007. The HFI is a multicolor focal plane which consists of 48 bolometers operated at $100 \mathrm{mK}$. Each bolometer is mounted to a feedhorn-filter assembly which defines one of six frequency bands centered between 100-857GHz. Four detectors in each of six bands are coupled to both linear polarizations and thus measure the total intensity. In addition, eight detectors in each of 3 bands $(143,217$, and $353 \mathrm{GHz})$ couple only to a single linear polarization and thus provide measurements of the Stokes parameters, $\mathrm{Q}$ and $\mathrm{U}$, as well the total intensity. The detectors are required to achieve a Noise Equivalent Power (NEP) at or below the background limit $\sim 10^{-17} \mathrm{~W} / \sqrt{\mathrm{Hz}}$ for the telescope and time constants of a few ms, short enough to resolve point sources as the 5 to 9 arc-minute beams move across the sky in great circles at $1 \mathrm{rpm}$. The bolometers are tested at $100 \mathrm{mK}$ in a commercial dilution refrigerator with a custom built thermal control system to regulate the heat sink with precision $<100 n K / \sqrt{H z}$. The $100 \mathrm{mK}$ tests include dark electrical characterization of the load curves, optical and electrical measurement of the thermal time constants and measurement of the noise spectral density from 0.01 to $10 \mathrm{~Hz}$ for up to 24 bolometers simultaneously.
\end{abstract}

Keywords: mm-wave sensors, bolometer, micro-machining, cosmic microwave background

\section{INTRODUCTION}

The ESA/NASA Planck Surveyor mission, to be launched in 2007, will perform a full sky survey of the mmwave universe. Here we report the status of the testing program for bolometer modules fabricated for the High Frequency Instrument (HFI) in the Planck spacecraft. Each bolometer module consists of a micromachined spiderweb bolometer, ${ }^{1}$ or pair of polarization sensitive bolometers (PSBs) ${ }^{2}$ mounted in a compact housing and complete with filters to attenuate RF power. The design and fabrication of the bolometer modules is described elsewhere. ${ }^{3}$ The bolometer modules are mounted to feedhorns with bandpass filters that define 6 bands, centered at $100,143,217,353,545$, and $857 \mathrm{GHz}$. The bands at 143,217 , and $353 \mathrm{GHz}$ are complemented with PSB modules. The bolometer, feedhorn and filter assembly, cooled to $100 \mathrm{mK}$, is mounted at the focal plane of a telescope with 9,7 and 5 arc minute resolution for the bands 100,143 , and $\geq 217 \mathrm{GHz}$ respectively. The spacecraft samples the sky by spinning at $1 \mathrm{rpm}$ at its orbit at the L2 Lagrange point. The response time, $\tau$, of all devices is fast enough to resolve point sources at the telescope resolution.

Acceptance tests of the bolometers consists of environmental and performance test in facilities at the Jet Propulsion Laboratory. ${ }^{4}$ A total of 31 bolometers have survived random vibration and shock tests. All bolometers have survived thermal cycling from ambient to cryogenic temperatures. The measured noise equivalent power (NEP) for all bolometers agrees with the calculated NEP. ${ }^{5}$ The measured $\tau$ meets requirement with $90 \%$ yield. However, $\tau \propto C$ is slower than expected due to excess heat capacity $C$. The measured excess $C$ is $\propto$ absorber area so we suspect it is residue from the fabrication process. We have completed acceptance testing

Further author information: (Send correspondence to W.H.)

W.H.: E-mail: Warren.A.Holmes@jpl.nasa.gov, Telephone: 18183545049 


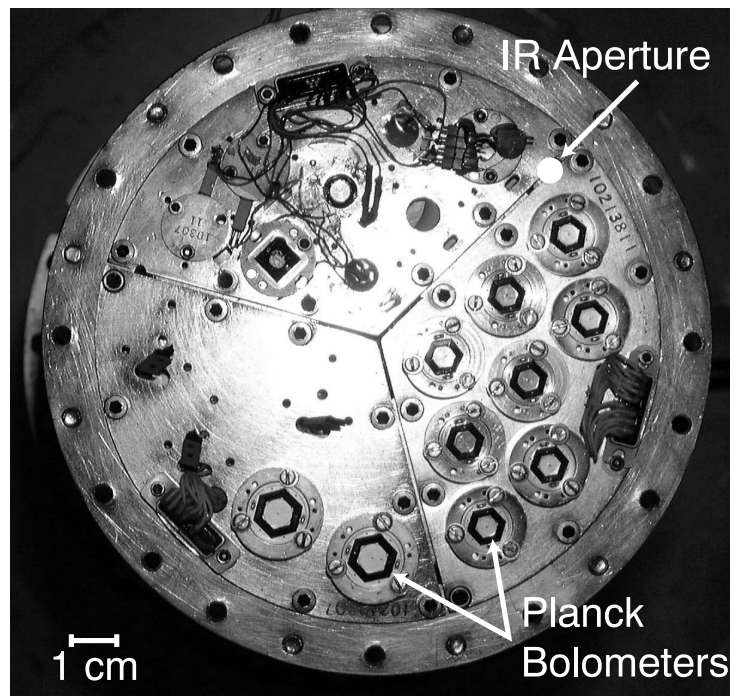

Figure 1. A photo of several bolometers mounted for testing.

of 32 cryogenic qualification module bolometers. Currently, we are testing noise and thermal performance of PSBs. Optical performance measurements, conducted at Caltech, are discussed elsewhere. ${ }^{2}$ Shortly, we will begin testing the Planck Flight Model (PFM) bolometers.

\section{TEST PROGRAM}

\subsection{Flight Acceptance Testing}

To ensure durability, all bolometers undergo two environmental tests before performance testing at 100mK. First, the bolometer modules are subjected to random vibration and shock tests at a local facility. The bolometers are transported to the test facility in a container hermetically sealed at the packaging laboratory to eliminate the chance of contamination and to protect the bolometers. At the vibration test facility, the transportation fixture is bolted to the shake table for test. The random vibration test level is $0.225 \mathrm{~g}^{2} / \mathrm{Hz}$ from $100-500 \mathrm{~Hz}$. This corresponds to a $3 \sigma$ acceleration of $45 \mathrm{~g}$. The shock test level is a $1000 \mathrm{~g}$ burst over $10 \mathrm{~ms}$. The random vibration and shock tests are applied along each orthogonal axis. Second, the bolometers are cycled between $300 \mathrm{~K}$ and $90 \mathrm{~K}$ at least three times in a custom built liquid nitrogen flow cryostat at JPL. When the bolometers are installed in or removed from the thermal cycling cryostat, filtered and ionized air is blown over the work area while the bolometers are moved from and to the transportation fixture to prevent contamination and damage due to electrostatic discharge.

The tests at $100 \mathrm{mK}$, are performed using an Oxford 25 dilution refrigerator insert equipped with 24 parallel voltage readout channels. ${ }^{4}$ The bolometers are mounted as shown in Figure 1 at the bottom of the dilution refrigerator insert, shown in Figure 2. During installation and removal from the dilution insert, filtered and ionized air is blown over work area as was done with the thermal cycling cryostat. Once all bolometers are mounted, the volume around the bolometers is hermetically sealed to prevent incidental contamination, for example, from vacuum pump oil.

The bolometers are current biased, $I_{b}$. The voltage, $V$, is readout using an amplifier chain with first stage JFET followers, as shown in Figure 3, and a warm preamplifier. We measure load curves at a series of temperatures $0.06<T<4 K$ with a DC bias and readout using only the DC coupled portion of the amplifier chain. These measurements are automated using Labview software and a National Instruments 6031E data acquisition card. The load curve data are analyzed using a spreadsheet. ${ }^{6}$ 


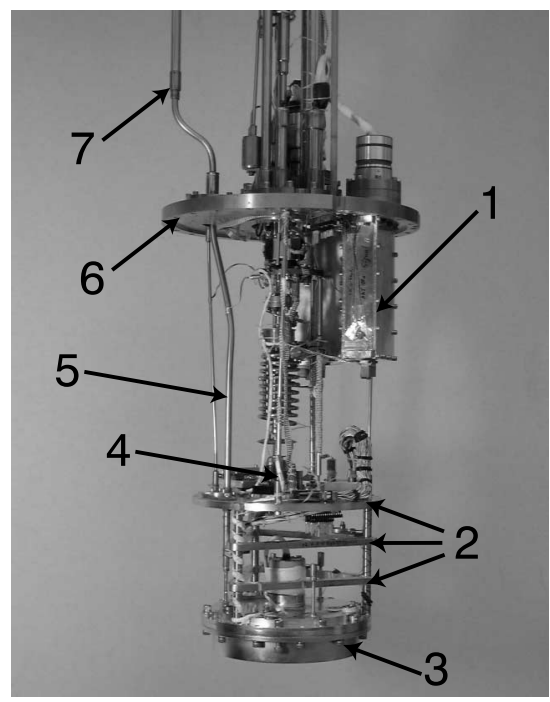

Figure 2. A photo of the dilution refrigerator insert used for testing at 100mK. The JFET box (1), thermal isolation stages (2), hermetic bolometer can (3), mixing chamber (4) and light pipe (5) are enclosed in a vacuum can sealed using indium wire to the insert flange (6) and immersed in liquid helium. Infrared filters or a brass plug can be changed while the insert is at $4 \mathrm{~K}$, at the threaded light pipe joint (7).

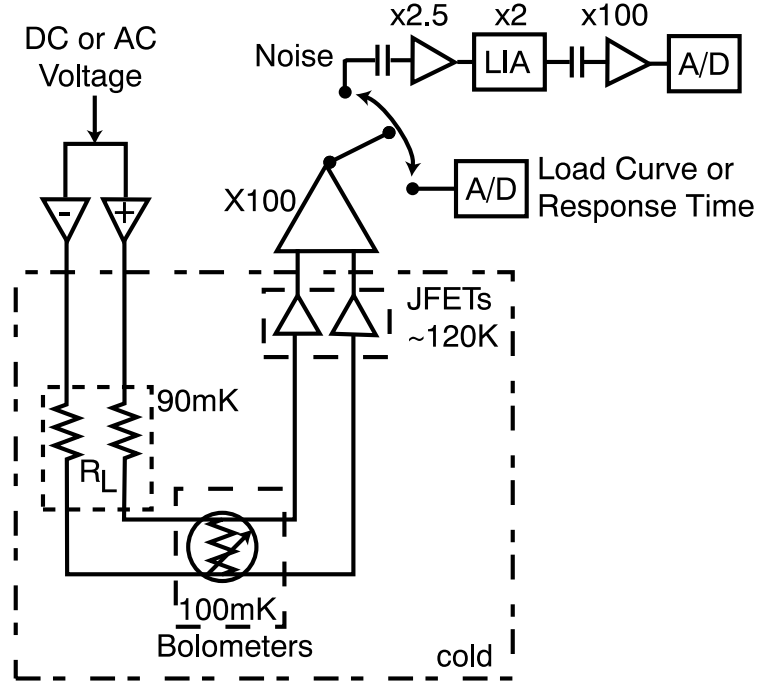

Figure 3. Amplifier chain used for measurement of bolometer performance at 100mK. A DC input voltage and a DC coupling is used for load curve and response time measurements. An AC input voltage, AC coupling and additional gain stages are used for the noise measurements. 


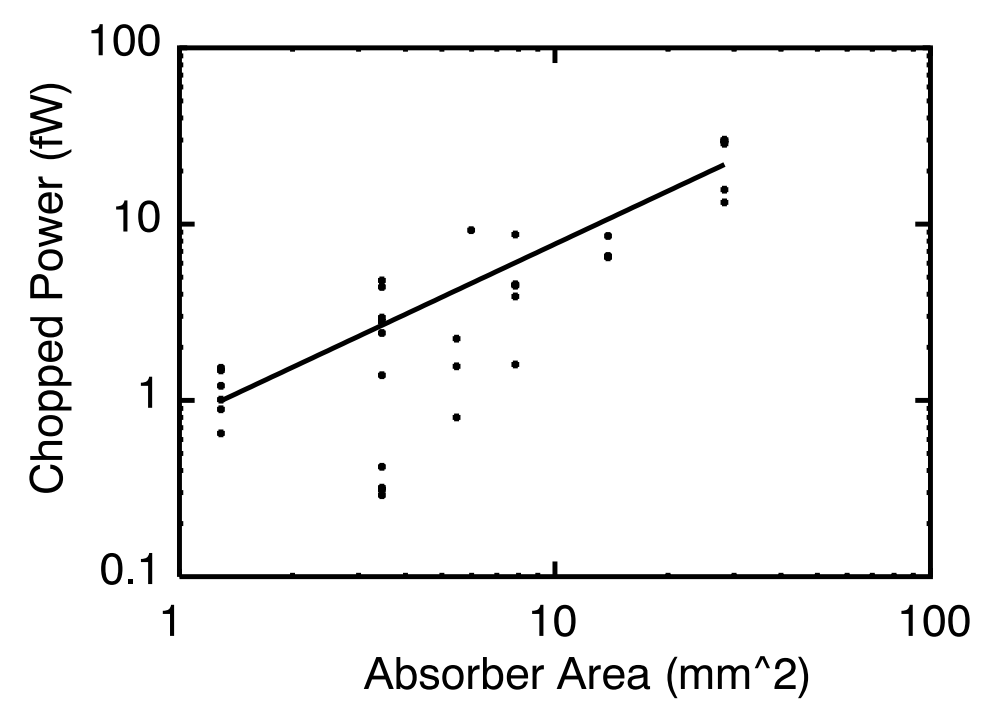

Figure 4. Absorber power as a function of effective mm-wave absorber area

We measure the bolometer noise with an anti-phase $\mathrm{AC}$ bias at $\sim 40 \mathrm{~Hz}$ applied to all bolometers simultaneously. The bolometer voltages are routed through additional AC coupled gain stages, synchronously demodulated, digitized, and recorded as time series in files, 5-10 minutes in length, using Labview software. The system gain is $\sim 6 \times 10^{4}$ and the bandwidth, post demodulation, is $\sim 8 \mathrm{~Hz}$. The data are sampled at $172 \mathrm{~Hz}$ to avoid aliasing harmonics of the AC bias into the noise power spectra. During the noise measurement, the heat sink temperature is precisely controlled, $<100 \mathrm{nK} / \sqrt{\mathrm{Hz}}$ at $f>16 \mathrm{mHz}$, using a multistage isolation and temperature regulation scheme ${ }^{7}$ with a dedicated set of 6 neutron transmutation doped (NTD) Ge thermometers and low noise readouts. Each thermometer is biased with a current modulated at $200 \mathrm{~Hz}$ and readout using cold JFET followers and a custom designed lockin amplifier mounted directly to the top flange of the cryostat insert. The temperature is fixed using a heater and commercial PID controller. Typically, the mixing chamber and one of the two thermal intercepts, shown in Figure 2 are regulated at fixed temperature. The temperature of the stages, the bolometer heat sink, regulation heater power and room temperatures are measured and recorded synchronously with the bolometer data. Usually, noise data are collected over night. The time series data files, $>1$ GByte total per night, are transferred to a different computer, deglitched, converted to a power spectrum, and averaged using IDL macros.

We measure the thermal response time of the bolometers, biased with a DC current, using a chopped infrared source. The bolometer signal is demodulated at the chop frequency using commercial lockin amplifiers and recorded as a function of frequency and DC bias. The infrared signal is generated and modulated using a commercial blackbody source and chopper. It is routed in a gold-plated thin walled stainless steel light pipe in the liquid helium through a hermetically sealed quartz window into the vacuum space of the dilution refrigerator unit and bolometer can. The light pipe attaches to the cold quartz window flange assembly via a threaded joint, shown in Figure 2. A brass plug is screwed into the light pipe during load curve and noise measurements and removed for optical response measurements. The plug and filters in the light pipe can be changed while the bolometers are at $100 \mathrm{mK}$ to adjust the IR signal if necessary. A second section of the light pipe extends from the quartz window flange through the vacuum space through a second set of infrared filters and attenuators at $\sim 100 \mathrm{mK}$ and sprays the infrared radiation into the bolometer can through a small aperture shown in Figure 1. The optical load, chopped and unchopped, on the bolometers is $<1 \mathrm{pW}$. The infrared exposure is surprisingly uniform within the bolometer can, $\sim 1 \mathrm{fW} / \mathrm{mm}^{2}$, as shown in Figure 4.

We also measure the response of the bolometer to a small square wave added to the DC bias. This is the so-called electrical time constant measurement. The amplifier is configured in exactly the same manner as used for the load curve measurement. The square waveform is generated in Labview software and output from the 
data acquisition card. Typically, the square wave is a $0.5 \mathrm{~Hz}$ ripple on the DC bias with an amplitude of $10 \%$ of the DC bias value. All bolometer output voltages are sampled simultaneously at a rate, per bolometer, of $2.5 \mathrm{kHz}$. A signal to noise ratio of $>100$ is obtained after about 20 averages.

\subsection{Qualification Testing}

After the flight acceptance tests, we select some bolometers for a set of more aggressive, qualification tests. About a half dozen bolometers are selected for one of two test sequences. One sequence is a more extreme version of the acceptance tests. First is a qualification thermal cycle test of 60 thermal cycles. After the thermal cycle test the bolometers are subject to a random vibration test at a level, $3 \sigma$ peak acceleration of $64 \mathrm{~g}$ or $90 \mathrm{~g}$, which is twice or four times that of the flight acceptance test for 1 minute along each axis. The resistance of the bolometer is measured frequently throughout these tests to monitor for failures. Once these tests are complete the bolometers are re-characterized at $100 \mathrm{mK}$.

The second sequence is an accelerated aging test. For this test, the bolometers are heated to $70^{\circ} \mathrm{C}$ at relative humidity of $85 \%$ for 1000 hours. This exposure simulates degradation of materials, such as the indium bump bonds and epoxy in the bolometer module assembly, which would occur over several years in storage. The resistance is monitored hourly using a current of $\sim 100 \mathrm{nA}$ applied for the reading interval of 1 second to avoid galvanic corrosion. These bolometers are retested at $100 \mathrm{mK}$ after the accelerated aging.

Two types of destructive tests are planned. The first is a random vibration 'shake and break' test. For the shake and break test, the bolometer is mounted and shaken along a fixed axis for one minute. If the bolometer is structurally intact by inspecting visually and by measuring electrical continuity across the NTD Ge leads, the level is increased by a factor $\sqrt{2}$. This process is repeated until the bolometer breaks. The second test is a fuse test where current is applied across the leads to the NTD Ge and increased until an open circuit is measured.

\section{TEST RESULTS}

\subsection{Environmental Acceptance Tests}

The bolometer survival yield through the acceptance vibration test is $>97 \%$. The yield through thermal cycling is $100 \%$. We have tested a few bolometers at $100 \mathrm{mK}$ before the vibration and thermal cycling and then after. We find no difference in performance within our experimental error of $\sim 3 \%$ due to the environmental testing.

\subsection{Load Curve Processing}

Detailed discussions of load curve processing are available in the literature ${ }^{6,8,9}$ Here we describe the steps we use to obtain data for our bolometers. First, we subtract the offset voltage and convert the externally applied voltage into the actual current bias at the bolometer. Then we fit the slope at low bias, to determine $R$ at all measurement temperatures. The resulting $\mathrm{R}(\mathrm{T})$ is fit to the form $R(T)=R_{\text {leads }}+R_{o} \exp \left(\sqrt{T_{g} / T}\right)$, where $R_{\text {leads }}$ is the lead resistance and $R_{o}$ and $T_{g}$ are standard fitting parameters for variable range hopping $(\mathrm{VRH})$ conduction. These fits are very good over the entire temperature range indicating that stray RF power or infrared power (for example from the infrared filters at $4 \mathrm{~K}$ in the light pipe) is negligible. The values of $R_{o}$ and $T_{g}$ are shown in Figure 5

Using the VRH fit parameters, we determine $T$ at each point in the load curve. Then we compute the thermal conductance, $G(T)=g_{o} T^{n}$ by fitting the bias power and temperature to the form $P=g_{o} /(n+1)\left(T^{n+1}+T_{o}^{n+1}\right)$. We have measured extremely small $G \sim 1 \mathrm{pW} / \mathrm{K}$ at $100 \mathrm{mK}$ for several contact engineering devices (CE) which had no spiderweb absorber. The fact that we can accurately measure $R_{o}, T_{g}$, and $G$ for devices with such a small value of $G$ sets an upper limit on the stray DC power of $1 \mathrm{fW}$. For the 545 and $857 \mathrm{GHz}$ bolometers with $G>200 \mathrm{pW} / \mathrm{K}$ non-ohmic effects in the thermometer material are important. ${ }^{10,11}$ For these devices we extrapolate the value of $\mathrm{G}(\mathrm{T})$ from $T>200 \mathrm{mK}$ to obtain the value at $100 \mathrm{mK}$. A compilation of all measured $G$ and corresponding $R_{\text {leads }}$ agrees with the Wiedemann Franz law $G \propto T / R_{\text {leads }}$.

Finally, we compute the bolometer responsivity, $S$, and NEP as a function of bias. In terms of V, G, the total load resistance $R_{L}=20 M \Omega$ and $\alpha=(1 / R) d R / d T=-1 / 2 \sqrt{T_{g} / T^{3}}, S$ is 


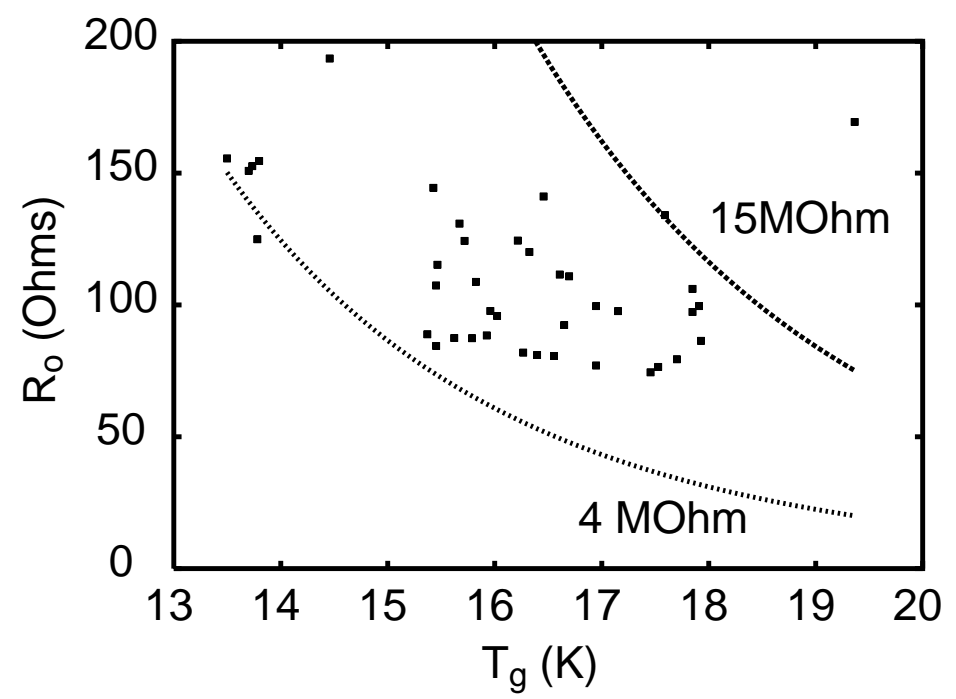

Figure 5. Measured values of $R_{o}$ and $T_{g}$ for all bolometers tested at $100 \mathrm{mK}$. The lines represent values of $R_{o}$ and $T_{g}$ for $R=4$ and $15 \mathrm{MOhm}$ close to the operating temperature $T \sim 130 \mathrm{mK}$.

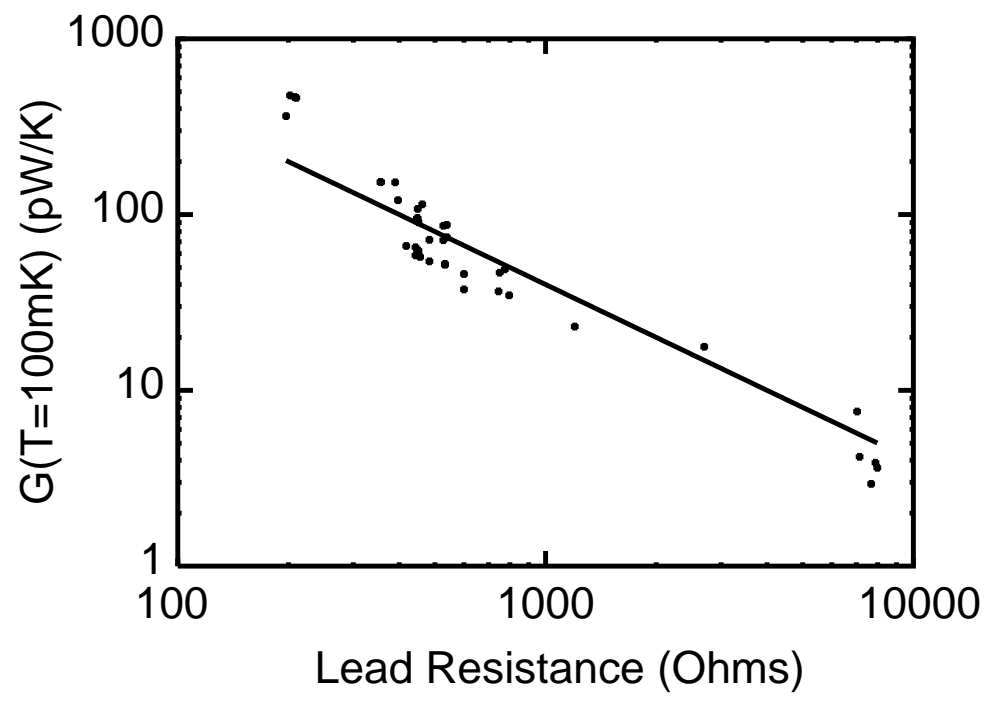

Figure 6. Measured thermal conductance at $100 \mathrm{mK}$ as a function of the lead resistance for several bolometers. The line is the $G$ predicted using the Wiedemann Franz law with no fitting parameters. 


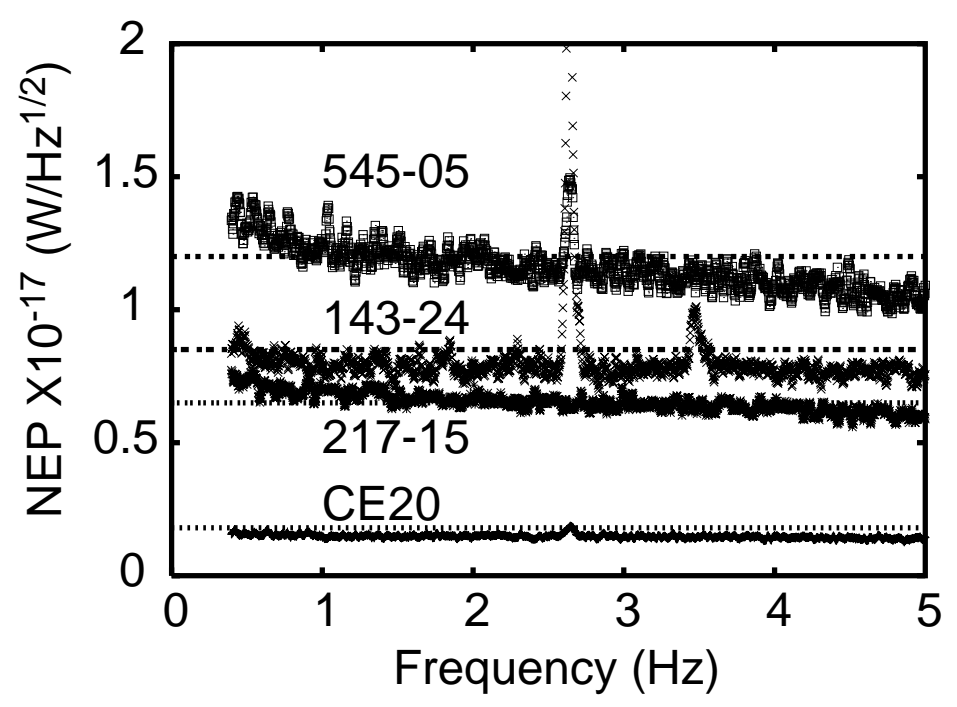

Figure 7. Measured NEP as a function of frequency for several bolometers. The dashed lines are the NEP calculated from the load curve data.

$$
S(f=0)=\alpha V /\left[(G-\alpha P)\left(1+R / R_{L}\right)\right]
$$

The NEP is

$$
N E P^{2}=\gamma_{1} 4 k T^{2} G+\gamma_{2} 4 k T R / S^{2}+e_{n}^{2} / S^{2}+N E P_{\text {excess }}^{2}
$$

where $\gamma_{1}$ and $\gamma_{2}$, typically of order 1 , account for non-equilibrium effects, ${ }^{5} e_{n} \sim 4 n V / \sqrt{H z}$ is the voltage noise of the amplifier and $N E P_{\text {excess }}$ is a generic excess noise term. The contribution from amplifier current noise is negligible.

\subsection{Bolometer Noise Measurement}

Noise power spectral density for typical bolometers over a wide range in sensitivity is shown in Figure 7 . The measured white noise is in good agreement with the noise calculated using Equation 2. The NEP of the CE devices is extremely low $N E P<2 \times 10^{-18}$. This measurement of low NEP on several CE devices validates our ability to measure well below the $N E P \sim 10^{-17} \mathrm{~W} / \sqrt{\mathrm{Hz}}$ specification for the Planck devices. Also, it constrains the background, non-optical, power drift over several minutes on any bolometer to $<10$ attoW. The voltage of bolometers with the highest $G$, in particular the 545 and $857 \mathrm{GHz}$ devices, when at optimum bias for lowest NEP, was near the saturation voltage at the input of the lockin amplifier. Even at a low bias, the bolometers satisfied the requirement for NEP. ${ }^{3}$

We have reduced the gain of the preamplifier to avoid this saturation effect. However, an additional complication is that, in fixed resistors, we measure excess noise at low frequencies at peak to peak voltages $>1 \mathrm{mV}$. Currently, we are debugging the cryogenic system and the electronics to eliminate this noise.

\subsection{Response Time Measurement}

The behavior $\mathrm{S}(\mathrm{f})$ can be modeled using a single pole thermal circuit as described in the literature. ${ }^{8}$ This model gives $\tau=C /(G-\alpha P)$, where $C$ is the heat capacity of the thermally isolated absorber and thermometer, and the term $\alpha P$ accounts for electro-thermal feedback (ETF). We calculate $\tau=1 / 2 \pi f_{3 d B}$ at the frequency, $f_{3 d B}$ where the bolometer response, normalized to the value at $f \sim 0$, has decreased by a factor $\sqrt{2}$. We have measured devices with $\tau<1 \mathrm{~ms}$ in our testbed. We calculate the heat capacity of each device from the value of 


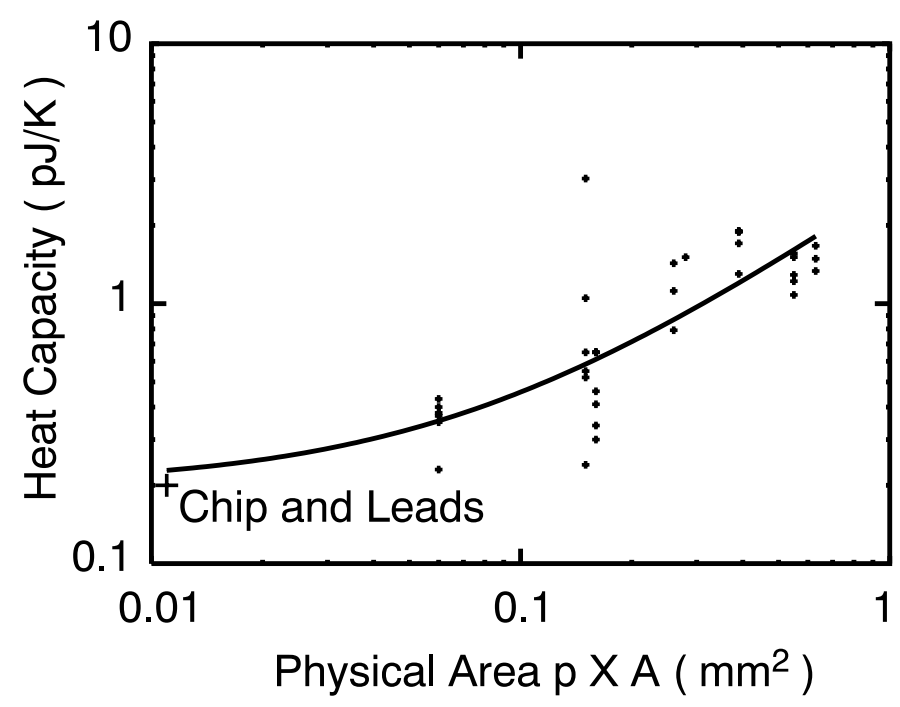

Figure 8. Bolometer heat capacities as a function of physical absorber area.

$\tau$ at low bias, where the ETF is negligible. In Figure 8, we fit the heat capacity as a function of physical area, $p A$ of the bolometer, which is the mm-wave absorber area, $A$, times the filling fraction, $p$ of the spiderweb. The intercept at $p A=0$ is fixed at the measured heat capacity of the NTD Ge chip and leads. The measured heat capacity of the chips and leads is in good agreement with the calculated value. ${ }^{3}$ However, any contribution that depends on area, such as the Si-N web or the absorber metalization, should be insignificant in comparison to the chip and leads. We suspect the excess heat capacity is contamination from the microprocessing.

\subsection{Qualification Tests}

Five devices were selected for the accelerated aging test. These devices were maintained at $70^{\circ} \mathrm{C}$ and in a relative humidity of $85 \%$ for 1100 hours. The resistance of 4 of 5 devices showed only a modest increase $<10 \%$ over the length of the test. Also we found no change in the weak humidity dependence of the resistance. One device measured open circuit immediately upon exposure to the accelerated aging environment. This failure is being analyzed.

The 4 surviving devices were retested at $100 \mathrm{mK}$. All measured characteristics of these bolometers, G, C and $\mathrm{R}(\mathrm{T})$ were unchanged. Furthermore, optical response, comparable to that before the accelerated aging, was measured indicating that the metalization of the spiderweb absorber was not effected by the aging test.

One bolometer was tested at a random vibration level four times the acceptance test level after acceptance testing. The electrical resistance, at room temperature, did not change during the test. After the vibration test, no mechanical damage was found on the spiderweb after the level test. Unfortunately, this bolometer failed because of excessive handling before it could be retested at $100 \mathrm{mK}$.

The survival of the bolometers in the accelerated aging test has eliminated degradation of bonded components over time as the cause of failed bolometers. The failure of any bolometer has been detected during the measurement of the resistance across the NTD Ge. This suggests that act of measuring the resistance, in fact, may contribute to the failures we have encountered. The fuse test will test this hypothesis.

\section{SUMMARY}

Flight acceptance testing at JPL of the CQM spiderweb bolometers for Planck HFI is complete. The bolometers have survived flight acceptance vibration and thermal cycle tests with a total yield $>90 \%$. The measured noise and response time of most of the CQM devices are better than required for the flight bolometers. We have 
measured values of $1<G<400 \mathrm{pW} / \mathrm{K}, \tau<1 \mathrm{~ms}$ and NEP lower than $2 \times 10^{-18} \mathrm{~W} / \sqrt{\mathrm{Hz}}$ at $100 \mathrm{mK}$ in our testbed. Bolometers for all unpolarized channels have been delivered to the University of Cardiff for optical tests with the focal plane hardware. Four bolometers have passed the accelerated aging test which simulated many years of 'shelf life'. The fabrication of CQM PSBs and the 100 GHz flight model bolometers is nearly complete and dark testing is underway. Several bolometers have been selected for qualification thermal cycle and vibration tests. Additional qualification testing of bolometers is planned, including a destructive 'shake and break' vibration tests and measurement of maximum readout current 'fuse' tests. Flight acceptance testing of flight model bolometers will begin this fall.

\section{ACKNOWLEDGMENTS}

This work was performed for the Jet Propulsion Laboratory, California Institute of Technology, sponsored by the National Aeronautics and Space Administration. We acknowledge helpful discussions with Bill Jones.

\section{REFERENCES}

1. P. Mauskopf, J. Bock, H. Del Castillo, W. Holtzapfel, and A. Lange, "Composite infrared bolometers with $\mathrm{Si}_{3} \mathrm{~N}_{4}$ micromesh absorbers," Appl. Opt. 36, pp. 765-771, 1997.

2. W. Jones, J. Bock, and A. Lange, "Polarization sensitive bolometers for Planck," in Proc. SPIE, 2002. submitted for publication.

3. M. Yun, T. Koch, J. Bock, W. Holmes, L. Wild, J. Mulder, A. Turner, C. Paine, and A. Lange, "Bolometric detectors for the Planck surveyor," in Proc. of SPIE, 2002. submitted for publication.

4. C. Paine, J. Bock, V. Hristov, and A. Lange, "A low noise, high thermal stability, 0.1K test facility for the Planck HFI bolometers," in Adv. Cryo. Eng., L. Summers, ed., 47, Plenum Press, New York, 2001.

5. J. C. Mather, "Nonequillibrium bolometer noise theory," Appl. Opt. 23, p. 3181, 1984.

6. J. C. Mather, "Bolometers: Ultimate sensitivity, optimization, and amplifier coupling," Appl. Opt. 23, p. 584, 1984.

7. J. Lipa, D. Swanson, J. Nissen, and T. Chui, "Lambda point experiment in microgravity," Cryogenics 34, pp. 341-347, 1994.

8. P. Richards, "Bolometers for infrared and millimeter waves," J. Appl. Phys 76, p. 1, 1994.

9. M. Griffin and W. Holland, "Bolometer optimization," Int. J. of IR and mm-Waves 9, p. 861, 1988.

10. S. Grannan, A. Lange, E. Haller, and J. Beeman, "Non-ohmic hopping conduction in doped germanium at T<1K," Phys. Rev. B 45, pp. 4516-4519, 1992.

11. D. McCammon, M. Galeazzi, D. Liu, W. Sanders, B. Smith, P. Tan, K. Boyce, R. Brekosky, J. Gygax, R. Kelley, D. Mott, F. Porter, C. Stahle, C. Stahle, and A. Szymkowiak, "1/f noise and hot electron effects in variable range hopping conduction," Phys. Stat. Sol. B 230, pp. 197-204, 2002. 